\title{
Kompozit bitirme ve cilalama işlemlerinin süt dişi pulpal sıcaklık değişimine etkisi
}

\author{
The effect of composite finishing and polishing procedures on primary tooth pulp \\ temperature change
}

\author{
Yıldırım Erdoğan, İhsan Furkan Ertuğrul
}

Gönderilme tarihi:10.09.2020

Kabul tarihi:25.11.2020

Öz

Amaç: Çalışmanın amacı, farklı bitirme ve cilalama sistemlerinin süt dişi intrapulpal sıcaklık artışı üzerindeki etkilerini, bir pulpal kan mikrosirkülasyon modeli yardımıyla değerlendirmektir.

Gereç ve yöntem: Çalışma için 10 sağlam insan üst ikinci süt azı dişi kullanıldı. 1 mm dentin kalınlığı kalacak şekilde standardize sınıf V kaviteler hazırlandı ve kompozit rezin ile restore edildi. Dişler, her grupta aynı 10 örnek olacak şekilde 6 gruba ayrıldı: Enhance bitim silindiri, Enhance PoGo cilalama silindiri, Sof-Lex spiral bitim lastiği, Sof-Lex spiral cilalama lastiği, Super-Snap Rainbow bitim diski, Super-Snap Rainbow süper-cilalama diski (n=10 her grup için). Bu 3 bitirme ve 3 cilalama ucu, 0,4 N güç, 10000 rpm hızda 10 sn boyunca düşük hızda el aleti ve mikromotor yardımıyla dişlerin üzerindeki restorasyonlara uygulandı. En yüksek sıcaklıklar ölçüldü ve not edildi.

Bulgular: En yüksek sıcaklık artışları, $2,3^{\circ} \mathrm{C}$ ile Enhance bitim silindiri ve Sof-Lex spiral bitim lastiği gruplarında, en düşük sıcaklık artışı ise $1,1^{\circ} \mathrm{C}$ ile Super-Snap Rainbow süper-cilalama diski grubunda görüldü. Super-Snap Rainbow süper-cilalama diski grubunun, her 3 bitim grubu ve Sof-Lex spiral cilalama lastiğine göre ortalama sıcaklık artışı anlamlı biçimde daha düşüktü. Hiç bir grup, geri dönüşümsüz hasara neden olan $5,6^{\circ} \mathrm{C}$ kritik pulpa içi sıcaklık artışı seviyesini geçmedi.

Sonuç: Bu çalışmanın koşulları altında, kuru uygulanan bitirme ve cilalama sistemlerinin süt dişi pulpaları üzerindeki sıcaklık artışı etkilerinin güvenli sınırlarda olduğu söylenebilir.

Anahtar kelimeler: Pulpal sıcaklık, süt dişleri, cilalama metodları, bitirme metodları, pulpal mikrosirkülasyon.

Erdoğan Y, Ertuğrul IF. Kompozit bitirme ve cilalama işlemlerinin süt dişi pulpal sıcaklık değişimine etkisi. Pam Tıp Derg 2021;14:556-564.

\begin{abstract}
Purpose: The purpose of this study was to evaluate the effect of different finishing and polishing systems on intrapulpal temperature of primary teeth assessed using a pulpal blood microcirculation model.

Materials and methods: 10 sound human maxillary second primary molars used. Standardized Class $\mathrm{V}$ cavity preparations were performed with dentin thickness of $1 \mathrm{~mm}$ and restored with composite resin. The teeth divided into 6 groups with same ten samples in each group: Enhance finishing cup, Enhance PoGo polishing cup, Sof-Lex spiral finishing wheel, Sof-Lex spiral polishing wheel, Super-Snap Rainbow finishing disc, Super-Snap Rainbow super-polishing disc ( $n=10$ per group). These 3 finishing and 3 polishing tips were applied to the restorations on the teeth with a force of $0.4 \mathrm{~N}$, at a speed of $10000 \mathrm{rpm}$ for 10 seconds with the help of a slowspeed handpiece and a micromotor. The highest temperature increase were measured and noted.

Results: The highest temperature rises was seen in the Enhance finishing cup and Sof-Lex spiral finishing wheel groups with $2.3^{\circ} \mathrm{C}$, and the lowest temperature measurement was in the Super-Snap Rainbow SuperPolishing Disc group with $1.1^{\circ} \mathrm{C}$. Super-Snap Rainbow super-polishing disc group demonstrated significantly lower mean values in comparison with all finishing groups and Sof-Lex spiral polishing wheel. None of the groups exceeded the critical intrapulpal temperature increase of $5.6^{\circ} \mathrm{C}$ that causes irreversible damage.

Conclusion: Under these study conditions, it can be suggested that the temperature increase effects of dry applied finishing and polishing systems on primary teeth pulps are within safe limits.
\end{abstract}

Key words: Pulp temperature, primary teeth, polishing methods, finishing methods, pulp microcirculation.

Erdogan Y, Ertugrul IF. The effect of composite finishing and polishing procedures on primary tooth pulp temperature change. Pam Med J 2021;14:556-564.

Yıldırım Erdoğan, Dr. Öğr. Üye. Pamukkale Üniversitesi, Diş Hekimliği Fakültesi, Pedodonti Ana Bilim Dalı, Denizli, Türkiye, e-posta: yldrmerdogan@hotmail.com (https://orcid.org/0000-0002-5054-1812) (Sorumlu Yazar)

İhsan Furkan Ertuğrul, Dr. Öğr. Üye. Pamukkale Üniversitesi, Diş Hekimliği Fakültesi, Endodonti Ana Bilim Dalı, Denizli, Türkiye, e-posta: furkanertugrul@gmail.com (https://orcid.org/0000-0001-7583-6679) 


\section{Giriş}

Restoratif diş hekimliğinde, hastaların artan estetik talepleri, kompozit rezinlerin kullanımının yaygınlaşmasını ve rezin teknolojisinde yüksek ivmeli bir ilerlemeyi sağlamıştır [1]. Geliştirilen özelliklerden biri, restorasyon yüzeylerinin daha pürüzsüz hale getirilmesidir [2]. Kompozit rezin restorasyonlarda pürüzsüz bir yüzey başarı için ayrılmaz bir gerekliliktir, pürüzlü yüzey bakterilerin tutunmasına, sekonder çürük gelişimine, aşınma kinetiğinin değişimine ve renk özelliklerinin kötü yönde etkilenmesine neden olabilir [3]. Kompozit rezinlerin yüzey pürüzlülüğü, iyi bir bitirme ve cilalama protokolü uygulanarak azaltılabilir. Bitirme ve cilalama işlemleri için karbit ve elmas frezler, ara yüz zımparaları, lastikler ve abraziv diskler rutin olarak kullanılan malzemelerdir [4].

Diş pulpası, sinirler, vasküler yapılar, lifler, interstisyel sıvılar, odontoblastlar, fibroblastlar ve diğer küçük hücresel bileşenleri içeren, dişin mezenşimal kökenli yumuşak dokusudur. Dentin dokusunun düşük termal iletkenliğine karşın pulpa, termal uyaranlara karşı oldukça hassastır [5]. Kavite preparasyonu [6], restoratif materyallerin polimerizasyonu [7], bitirme/ cilalama [8] gibi birçok dişsel işlem, pulpa sıcaklığını artırma potansiyeline sahiptir. İntrapulpal sıcaklıktaki artış, bu dokunun sağlığına önemli ölçüde zarar verebilir. Rhesus maymunları üzerinde yapılan klasik bir çalışma, $5,6^{\circ} \mathrm{C}$ 'lik bir sıcaklık artışının deneklerin $\% 15^{\prime}$ inde geri dönüşümsüz pulpitise yol açtığını, artış $11,1^{\circ} \mathrm{C}$ olduğunda $\% 60,16,6^{\circ} \mathrm{C}$ olduğunda ise \%100'e çıktığını göstermiştir [9]. Pulpal sıcaklık artışı ile ilgili birçok araştırma bu çalışmayı işaret etmektedir ve yaygın olarak kabul edilen tehlikeli pulpal sıcaklık artışının $5,6^{\circ} \mathrm{C}$ olduğu söylenebilir [10-13].

Aşınma, materyalin bir yüzeyden mekanik işlemlerle küçük parçacıklar şeklinde çıkarıldığı kümülatif yüzey hasarı fenomeni olarak tanımlanır. Bitirme ve cilalama malzemeleri ve işlemleri, dental restoratif materyal yüzeylerinin özel, seçici ve kontrollü aşınmasını sağlamak üzere tasarlanmıştır. Aşınma mekanizması, materyalin uzaklaştırılması veya yer değiştirmesi ile enerjinin aktarımasıdır [14]. Enerji aktarımı, aşınma bölgesinde yoğunlaşan ısıya dönüşür, bu da aynı bölgede keskin bir sıcaklık artışına yol açar [15]. Sıcaklık artışı, pulpa vitalitesini negatif etkileyebilir.
Çalışmamızın amacı, 3 farklı firmanın ürettiği bitirme ve cilalama sistemlerinin, süt dişi pulpal sıcaklık artışı üzerindeki etkilerinin bir pulpal kan mikrosirkülasyon modeli (PKMM) yardımıyla belirlenmesidir.

\section{Gereç ve yöntem}

$\mathrm{Bu}$ çalışmanın gerçekleştirilmesi için Pamukkale Üniversitesi Tıbbi Etik Kurulu'ndan Etik Kurulu'ndan onay alınmıştır.

Çalışmada, fizyolojik kök rezorpsiyonu nedeniyle çekimi yapılmış, çürük veya akut travma nedeniyle hasarı bulunmayan 10 adet üstikinci süt azı diş kullanıldı. Dişlerin çekimi yapıldıktan sonra ISO standartları gereği akan su altında yüzey dokuları ve kan uzaklaştıııldı [16] ve \%10 formalin içinde 2 hafta dezenfekte edilerek, kullanılıncaya kadar distile su içinde muhafaza edildi [17]. Kullanılmadan önce dişlerin kökleri, mine-sement sınırının $2 \mathrm{~mm}$ altından dişlerin uzun eksenine dik şekilde su soğutmalı el aleti ve fissür frez yardımı ile düzeltildi. Pulpa odasının apikal kısmı da benzer şekilde genişletildi, pulpal doku artıkları ekskavatör yardımı ile retrograd olarak nazikçe uzaklaştırıldı, bölgeye $\% 5,25$ sodyum hipokloritli pamuk pelet $1 \mathrm{dk}$ tatbik edildi, sonrasında distile su ile yıkandı ve pamuk peletle kurutuldu. Dişlerin bukkal yüzlerine, pulpa odasına yaklaşık $1 \mathrm{~mm}$ mesafe kalacak şekilde, su soğutmalı olarak yüksek hızla çalışan el aleti ve fissür frezle, $3 \times 2 \times 2 \pm 0,5 \mathrm{~mm}$ boyutlarında retantif olmayan kaviteler açıldı. Kavite tabanı ve pulpa odası arasındaki kalan dentin kalınlığı, 0,05 mm'ye kadar hassas kadranlı bir kumpas (Yates-Motloid Co., Chicago, IL, USA) ile belirlendi.

Sıcaklık ölçümlerini elde etmek için, ilk olarak Ertuğrul ve ark.'nın [18] çalışmasında tasarlanmış ve tarif edilmiş pulpal kan mikrosirkülasyon modeli kullanıldı. Termokupl telini yerleştirebilmek içinbir elmas frez ile dişlerin lingual yüzeylerinde küçük bir delik açılarak erişim sağlandı. K tipi bir termokupl teli (TT-K-30-SLE; Omega Engineeringlnc, Stanford, CT, USA) pulpa odasına konuldu ve silikon ISı transfer bileşiği kullanılarak (ZMSTG2; Zalman Tech Co. Ltd, Dongan-gu, South Korea) dişin aksiyal duvarına yerleştirildi.

Termokupl telini sabitlemek ve erişim boşluğu ile tel arasındaki boşluğu kapatmak için, 6 . nesil 
iki aşamalı self-etch bonding sistem (Clearfil SE Bond, Kuraray®, Tokyo, Japan) ve akışkan bir kompozit (Filtek Ultimate Flowable Restorative, 3M ESPE, St. Paul, MN, USA) kullanılarak, $1470 \mathrm{~mW} / \mathrm{cm}^{2}$ ışık yoğunluğuna sahip bir LED ışık cihazıyla (Elipar Deep Cure-S, 3M ESPE, St. Paul, MN, USA) sertleştirildi. Termokupl telinin diğer tarafı, pulpa odasındaki sıcaklık değişikliklerini izlemek için kişisel bir bilgisayara bağlanan dört kanallı bir veri kaydediciye (DT3891G; CEM, Shenzhen, PRC) bağlandı.

Çalışma modeli için platform olarak 3 mm kalınlığında alüminyumdan özel olarak tasarlanmış, termal iletkenliği $20^{\circ} \mathrm{C}$ 'de $222 \mathrm{~W} /$ mK olan bir plaka (1050-H14, ISO 9001: 2000, Bozhong Group, Shangai, China) kullanıldı. Plakanın tabanı, pulpa odasına distile su giriş ve çıkışını sağlamak için 2 defa delindi. Her diş örneği deliklere yerleştirildi ve her bir termal testte siyanoakrilat yapıştırıcısı kullanılarak tutturuldu. Bir çift paslanmaz çelik $\mu$ boru (25G, Hayat Medical Co., Lot \# 8696569000777, Istanbul, Turkey) deliklere yerleştirildi ve siyanoakrilat yapıştırıcı kullanılarak tabana tutturuldu. Rezervuar olarak $37^{\circ} \mathrm{C}$ standart fizyolojik sıcaklık seviyesinde ısıtılmış damıtılmış su ile doldurulmuş bir dijital su banyosu (WB-11; Daihan, Wonju, South Korea) kullanıldı. Bir dijital infüzyon akış ölçer (Outlook 100ES, B. Braun Medical, Inc, Bethlehem, PA, USA), akış hızını 0,026 mL/dk'ya stabilize etmek için sisteme entegre edildi. Bu rakam, bir pulpa odası içindeki kan akışının fizyolojik değeri olarak kabul edilir [19].

Sistem içindekisıcaklık kaybını telafi etmek ve giren suyun $37^{\circ} \mathrm{C}$ sıcaklığa ulaşmasını sağlamak için bir silikon ısı transfer bileşiği kullanılarak ana malzemenin altına spiral şeklinde bir bakır boru takıldı. İçeri akış ısı dengeleyici aparat, diğeriyle aynı akış hızına ayarlanan başka bir dijital infüzyon akış ölçere (Outlook 100ES, B. Braun Medical, Inc.) bağlanarak, damıtılmış su sirkülasyon sistemine entegre edildi. Restorasyonların bukkal bölgesine bitirme ve cilalama materyalleri uygulanarak, pulpa içi sıcaklık değişimleri ölçüldü.

Deneysel gruplar şu şekilde oluşturuldu; Enhance Bitim Silindiri (EBS), Sof-Lex Spiral Bitim Lastiği (SSBL), Super-Snap Rainbow Bitim Diski (SRBD), EnhancePoGo Cilalama Silindiri (EPCS), Sof-Lex Spiral Cilalama Lastiği (SSCL), Super-Snap Rainbow Süper-Cilalama Diski (SRCD) (Tablo 1, Resim 1). Süt dişlerinin bukkal yüzlerine açılan kavitelere $3 \mathrm{M}^{\mathrm{TM}}$ Filtek $^{\mathrm{TM}}$

Tablo 1. Çalışmada kullanılan bitirme ve cilalama materyalleri ve çalışmanın detayları

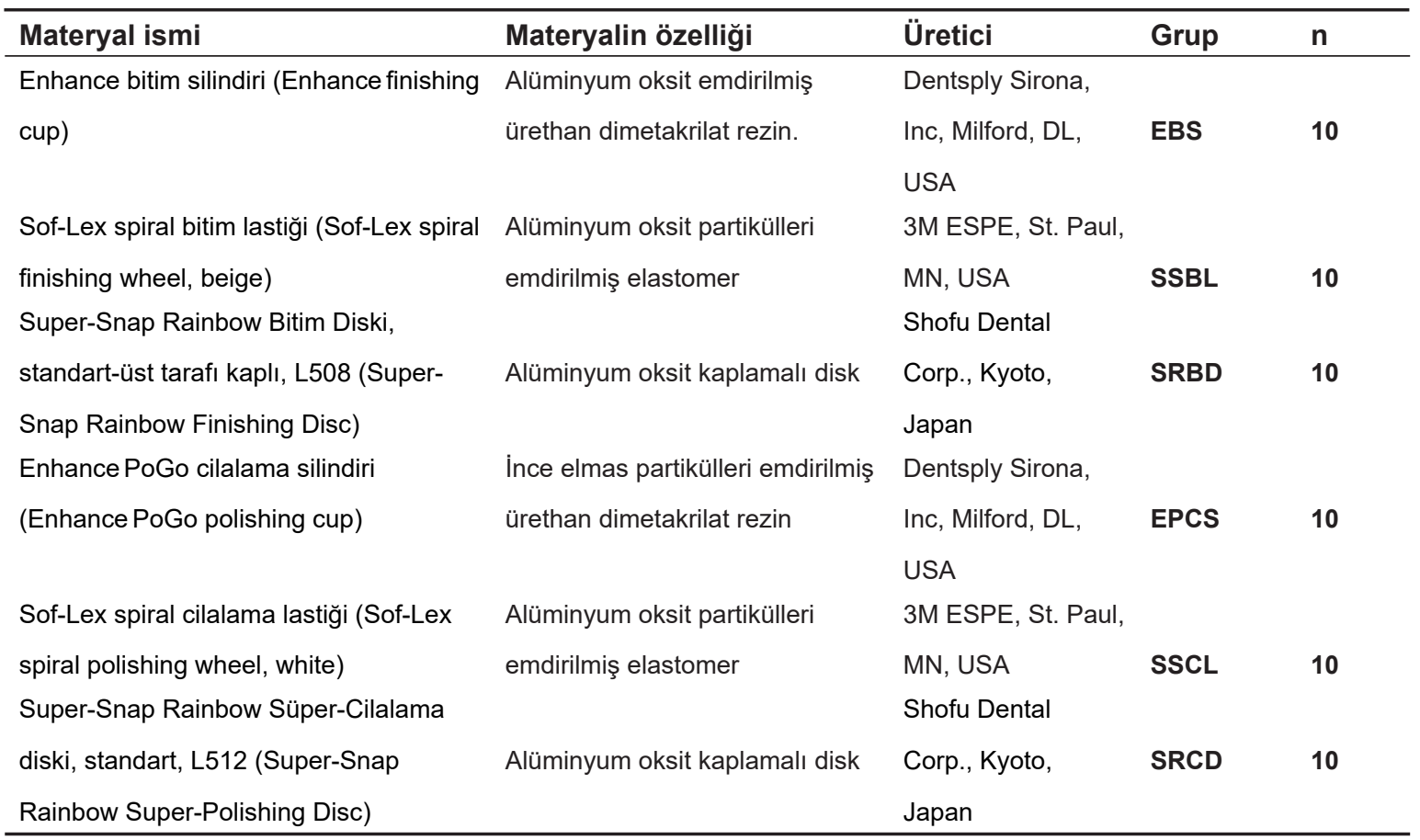




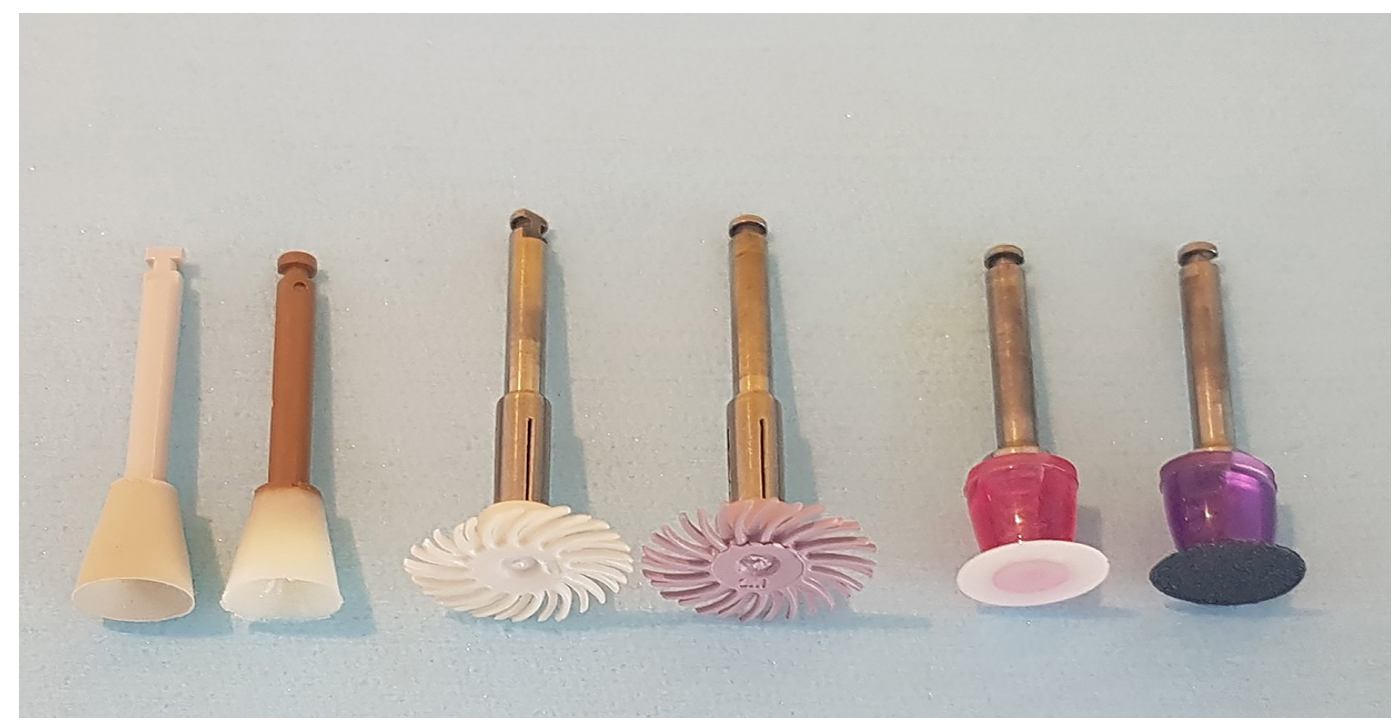

Resim 1. Çalışmada kullanılan bitirme ve cilalama uçları

(soldan sağa doğru sırasıyla); EnhancePoGo Cilalama Silindiri (EPCS), Enhance Bitim Silindiri (EBS), Sof-Lex Spiral Bitim Lastiği (SSBL), Sof-Lex Spiral Cilalama Lastiği (SSCL), SuperSnap Rainbow Süper-Cilalama Diski (SRCD), Super-Snap Rainbow Bitim Diski (SRBD)

Bulk Fill Posterior Restorative (A2) rezin kompozit materyal uygulandı ve 20 sn boyunca $1470 \mathrm{~mW} / \mathrm{cm}^{2}$ gücünde ışık veren LED cihazı Elipar Deep Cure-S ile polimerize edildi.

Düşük hızla çalışan bir el aletine (Mastermatic Lux M20L, KaVo Dental GmbH, Biberach, Germany) bağlanmış elektrikli bir mikromotor (Intra Lux KL701, KaVo Dental GmbH, Biberach, Germany) 40 g'lık (0,4 N) sabit bir ağırlığa bağlı bir tutucu üzerine monte edildi. Mikromotor, 10,000 rpm hıza ayarlandı ve bitirme-cilalama uçları 10 sn boyunca 0,4 $\mathrm{N}$ kuvvetle süt dişinin bukkal bölgesine yapılan kompozit restorasyona aynı açı ile temas ederek çalıştıııldı (Resim 2). Her bitirme veya cilalama ucu test edildikten ve pulpal sıcaklık ölçümleri yapıldıktan sonra bir sond yardımıyla kavitedeki kompozit çıkarıldı ve tekrar kompozit uygulandı. Toplam 6 materyal test edilip, sıcaklık değişimleri kaydedildikten sonra diş yerinden çıkarılıp, farklı bir diş daha önce bahsedilen şekilde modele adapte edildi ve test işlemleri tekrarlandı. Başlangıç sıcaklığı ile en yüksek sıcaklık arasındaki sıcaklık değişimi ölçüldü ve bir yazılım yardımıyla kaydedildi (Multiple Data Logger, AzeoTech Inc., Ashland, Oregon, USA).

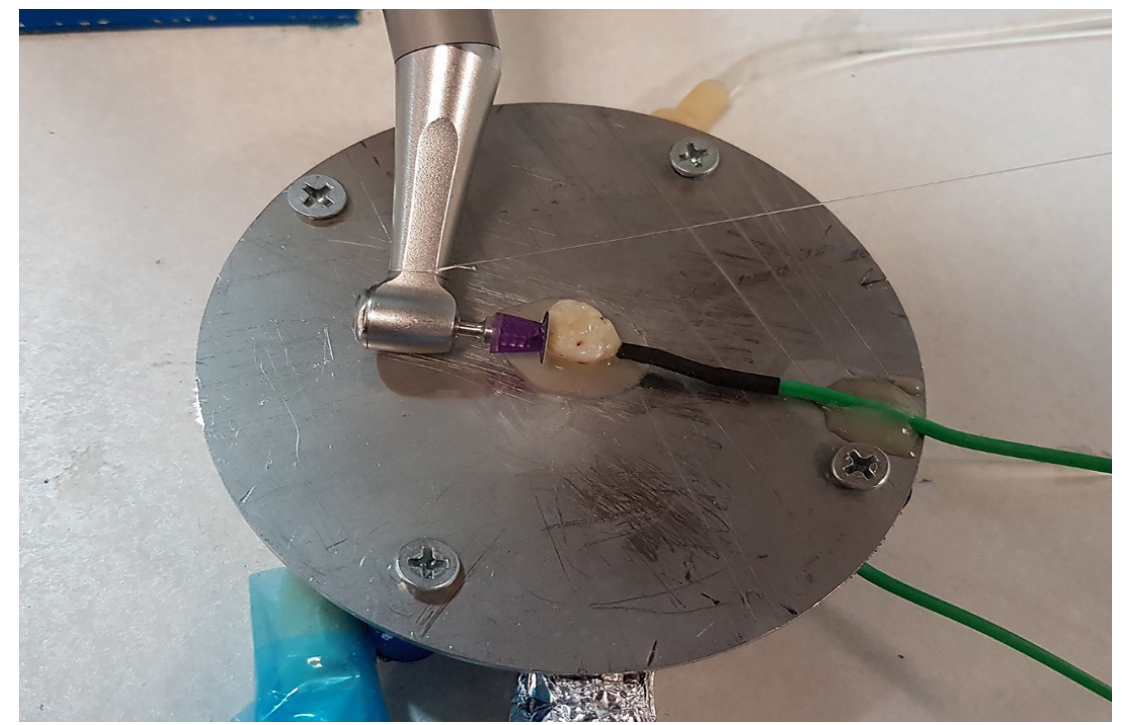

Resim 2. Pulpa kan mikrosirkülasyon model kurulumunun fotoğrafı 
İstatistiksel analizde verilerin normal dağılıma uygunluğu Shapiro-Wilk testi ile incelendi. Parametrik test varsayımları sağlandığında bağımlı grup farklılıkların karşılaştırılmasında İki Eş Arasındaki Farkın Önemlilik testi ve Tekrarlı Ölçümlerde Varyans analizi (post hoc: Bonferroni yöntemi), parametrik test varsayımları sağlanmadığında ise Wilcoxon Eşleştirilmiş İki Örnek testi ve Friedman testi (post hoc: Bonferroni düzeltmeli Wilcoxon Eşleştirilmiş İki Örnek testi) kullanıldı. (SPSS version 23.0, IBM Corporation, Armonk, New York, USA). Analizlerde, $p<0,05$ istatistiksel olarak anlamlı kabul edildi.

\section{Bulgular}

10 sn kuru uygulama işlemlerinden sonra, en yüksek sıcaklık ölçümü $2,3^{\circ} \mathrm{C}$ ile EBS ve SSBL gruplarında, en düşük sıcaklık ölçümü ise $1,1^{\circ} \mathrm{C}$ ile $S R C D$ grubunda görüldü. $S R C D$ grubu, SSCL grubuna ve SRBD grubuna göre istatistiksel olarak anlamlı biçimde düşük ortalama sıcaklık artışı gösterdi $(p<0,05)$. Hiçbir grup, kritik sıcaklık değeri olan $5,6^{\circ} \mathrm{C}$ 'nin üstüne çıkmadı (Tablo 2).

Tablo 2. Deney gruplarına ait sıcaklık artışı ortalama değerleri ve karşılaştırma

\begin{tabular}{|c|c|c|c|c|c|}
\hline & \multicolumn{2}{|c|}{ Bitirme } & \multicolumn{2}{|c|}{ Cilalama } & \multirow[b]{2}{*}{$p$} \\
\hline & Ort. \pm Std. Sap. & Med (min-maks) & Ort. \pm Std. Sap. & Med (min-maks) & \\
\hline \multirow[t]{2}{*}{ Enhance/PoGo silindir (1) } & $1,85 \pm 0,34$ & $1,85(1,3-2,3)$ & $1,65 \pm 0,3$ & $1,6(1,3-2,2)$ & $0,239(z=-$ \\
\hline & (EBS) & & (EPCS) & & $1,177)$ \\
\hline \multirow[t]{2}{*}{ Sof-Lex spiral lastik (2) } & $1,9 \pm 0,25$ & $1,8(1,6-2,3)$ & $1,82 \pm 0,18$ & $1,85(1,5-2,1)$ & $0,466(t=0,76)$ \\
\hline & (SSBL) & & (SSCL) & & \\
\hline Super-Snap Rainbow & $1,69 \pm 0,25$ & $1,8(1,3-2)$ & $1,39 \pm 0,3$ & $1,35(1,1-2,1)$ & $0,009^{*}$ \\
\hline disk (3) & (SRBD) & & (SRCD) & & $(\mathrm{t}=3,308)$ \\
\hline$p$ & \multicolumn{2}{|c|}{$0,293(F=1,316)$} & \multicolumn{2}{|c|}{$0,007^{*}\left(X^{2}=9.892\right)(2-3)$} & \\
\hline
\end{tabular}

F: Tekrarlı ölçümlerde varyans analizi, X2:Friedman Testi, z: Wilcoxon eşleştirilmiş iki örnek testi, t: İki eş arasındaki farkın önemlilik testi, Ort: Ortalama, Std.Sap.: Standart sapma, Med: Medyan, min: minimum değer, maks: maksimum değer

\section{Tartışma}

Pürüzsüz bir yüzey, rezin restoratif işlemlerin sadece estetik görünümü için değil, aynı zamanda yumuşak dokuların sağlığı ve restoratif ara yüzlerin marjinal bütünlüğü için de ana hedefi olmuştur. Yüzey pürüzlülüğü, bakteriyel plakta ileri derecede artışa neden olarak, çürük ve periodontal enflamasyon riskini de arttırmaktadır [20].

Restorasyonlarda bitirme işlemi, marjinal düzensizliklerin giderilmesini, anatomik konturların belirlenmesini ve "diş yapısının ve restoratif materyalin birleşme noktasında fazla restoratif materyalin çıkarılmasını ve pürüzsüz, düzgün, iyi adapte edilmiş bir kavite-dış yüzey kenarı oluşturmak için çeşitli tekniklerinin uygulanması" anlamına gelen, "marjinasyon" denen spesifik adımı da kapsayan işlemlerin bütünüdür. Cilalama ise, restorasyon yüzeyinden küçük çizikleri gidermek ve pürüzsüz, ışığı yansıtan bir parlaklık elde etmek için bitirme işleminden sonar gerçekleştirilen işlemdir. En az mikroskopik çiziklere ve sapmalara sahip homojen bir yüzey üretmeyi amaçlamaktadır [14].

Teknik olarak 4 ana aşınma mekanizması tanımlanmıştır; adezyon, abrazyon, yüzey yorgunluğu ve tribokimyasal reaksiyonlar. Bitirme ve cilalama işlemleri ile meydana gelen aşınmanın daha çok abrazyon tipinde olduğu bildirilmektedir. Yüzeyin ve yüzeyaltının fiziksel ve kimyasal özelliklerinin değiştiği bu mekanik deformasyon, yüksek oranda lokalize ISI üretimine neden olur [14].

Sıcaklık artışının diş ve çevre dokular üzerindeki etkisi üzerine farklı çalışmalar mevcuttur. Zach ve Cohen'in [9] hayvan çalışmasında $5,6^{\circ} \mathrm{C}$ sıcaklık artışının, dişlerin $\% 15$ 'inde pulpitis ve pulpa nekrozuna yol açtığı bildirilirken, Baldissara ve ark. [21] $11,2^{\circ} \mathrm{C}$ sıcaklık artışının pulpaya zarar vermediğini belirtmiştir. Pohto ve Schenin [22], diş pulpasına zarar verebilecek kritik sıcaklığın $42,5^{\circ} \mathrm{C}$ olduğunu bulmuşlardır. Schubert [23], pulpal 
fibroblastlar için kritik limitin $41,5^{\circ} \mathrm{C}$ olduğunu bildirmiştir. Eriksson ve Albrektsson [24], $10^{\circ} \mathrm{C}$ sıcaklık artışının kemik rezorpsiyonu ve diş ankilozuna neden olduğunu göstermiştir. Pulpal sıcaklık artışı ile ilgili çalışmaların birçoğu, Zach ve Cohen'in çalışmasına gönderme yapmaktadır [10-13] ve $5,6^{\circ} \mathrm{C}$ pulpal sıcaklık artışının kritik olduğu yönünde genel bir kanı olduğu söylenebilir.

Çalışmada, süt azı dişine kompozit rezin yerleştirildikten sonra bir sond ucu kompozitin köşesine batırılarak küçük bir girinti oluşturulmuş, bu şekilde kompozit ışıkla sertleştirilmiş, bitirme veya cilalama uçlarından biri test edilmiş ve sıcaklık ölçümü yapıldıktan sonra kompozit restorasyon, sond ucunun önceden oluşturulmuş girintiye yerleştirilerek kaviteden çıkarılmış, sonraki bitirme/cilalama materyalinin test edilmesi için tekrar aynı yöntemle kompozit rezin yerleştirilmiştir. Materyallerin hepsi denendikten sonra süt dişi modelden çıkarılmış, yeni bir süt dişi adapte edilmiş ve tüm işlemler tekrar uygulanmıştır. Dişlerin anatomik ve fizyolojik yapıları birbirinden farklıdır, sert doku kalınlıkları, dentin tübüllerinin çapları ve miktarları değişkendir. Dişsel işlemlerin pulpal sıcaklık artışına etkisini inceleyen bazı in vitro çalışmalar, tüm deneyi tek bir diş üzerinde yaparak standardizasyon sağlamayı amaçlamışlardır [21-25]. Deneyin geçerlilik ve güvenirliğini arttırmak için çalışmada 10 adet üst süt II. azı diş kullanılmış ve her bitirme/cilalama ucu kullanımından önce kompozit restorasyon değiştirilmiştir. Böylelikle her seferinde, aşındırma işlemlerine uğramamış yeni kompozit rezin ile ölçümler yapılmış, ayrıca materyallerin tümü, her dişe uygulanarak standardizasyon sağlanmıştır. Bitirme/cilalama uçları, farklı dişte işlem yapılacağı zaman değiştirilmiştir.

Literatürde, kavite preparasyonu [26-29], rezin polimerizasyonu [30-34], ışık kaynakları $[12,35,36]$ ve diş beyazlatma işlemlerinin [13, 37-39] pulpal sıcaklık artışı ile ilişkisini inceleyen çalışmaların yanı sıra, yüzey bitirme ve cilalama işlemlerinin pulpal sıcaklık artışına olan etkisini inceleyen birkaç çalışma mevcuttur [18, 4042]. Bitirme-cilalama işlemlerinin süt dişi pulpal sıcaklık değişimine etkisini inceleyen herhangi bir çalışmaya rastlanmamıştır.

Pulpal sıcaklık artışı üzerine yapılan benzer çalışmalarda, genellikle, termokupl, pulpa odası içinde termal bağlantı maddeleri kullanılarak kavite duvarına komşu bölgeye tutturulmuş, diş örneği, mine-sement birleşimine kadar, mikrosirkülasyon olmadan önceden $37^{\circ} \mathrm{C}$ 'ye kadar ısıtılmış su banyosuna gömülmüştür [30, $41,43]$. Çalışmamızda uyguladığımız PKMM'de ise, pulpal kapiller kan dolaşımını taklit edebilmek için bir içeri akış ısı dengeleyicisi eklendi. Böylece rezervuardan $37^{\circ} \mathrm{C}$ ile yola çıkan, ancak dişe ulaştığında sıcaklık kaybetmiş olduğu görülen suyun sıcaklığı dengelendi. Pulpa odasına su akışını sürdürmek için, sisteme otomatik bir infüzyon pompası entegre edildi ve mikrodolaşım, 0,026 $\mathrm{mL} / \mathrm{dk}$ hıza ayarlandı; bu değer, pulpal kan akışının in vivo akış hızıdır.

Çalışmada, bitirme ve cilalama malzemelerinin kuru ortamda çalışması önerilen malzemeler arasından seçildi, böylece su veya lubrikant özellikteki maddelerin soğutma özelliğinin, pulpal sıcaklık değerleri üzerindeki olası etkisi devre dışı bırakıldı. Enhance Bitim Silindiri ve EnhancePoGo Cilalama Silindiri'nin 10,000-15,000 rpm devirlerinde, hafif basınç ile çalışılması önerilmektedir [44]. Super-Snap Rainbow Bitim Diski ve Süper-Cilalama Diski için üretici tavsiyesi, 10,000-12,000 rpm hızda 0,3-0,6 N kuvvetle kullanılmasıdır [45]. Sof-Lex Spiral Bitirme ve Cilalama Lastikleri için ise üretici tavsiyesi, düşük hızlı el aleti ile 10,00020,000 rpm ile kullanılmasıdır ve basınç için herhangi bir öneri yoktur [46]. Yüzey bitirme ve cilalama işlemlerinin pulpal sıcaklık artışına olan etkisini inceleyen az sayıda çalışma içinde, Briseno ve ark. [42] 4,000-10,000 rpm ve 2 dk işlem süresi, Mank ve ark. [40] 40,000 rpm, 0,4 $\mathrm{N}$ basınç ve 10 sn işlem süresi, Ertuğrul ve ark. [18] 20,000 rpm, 0,4-0,8 $\mathrm{N}$ basınç ve $10 \mathrm{sn}$ işlem süresi ile farklı materyalleri uygulamışlardır. Bu çalışmalardan ikisi, farklı soğutma koşullarını da test etmişlerdir [40, 42]. Bu çalışmalar da değerlendirilerek, çalışmamızdaki tüm malzemeler için üretici firma tarafından önerilen devir sınırlarının içinde kalan 10,000 rpm, 0,4 N basınç ve 10 sn işlem süresi tercih edildi ve kuru ortamda uygulandı.

Cilalama ve bitim işlemlerinin ortalama sıcaklıklarına bakıldığında, her bir firma için cilalamanın, bitim işlemine göre daha az pulpal sıcaklık artışına yol açtığı, ancak istatistiksel olarak sadece Super-Snap Rainbow SüperCilalama diski $\left(1,39 \pm 0,3^{\circ} \mathrm{C}\right)$ ile Super-Snap 
Rainbow Bitim Diski $\left(1,69 \pm 0,25^{\circ} \mathrm{C}\right)$ arasındaki farkın anlamlı olduğu görüldü. Cilalama uçlarında emdirilmiş ya da kaplanmış olarak kullanılan Alüminyum oksit ya da elmas partiküllerinin çaplarının, bitim uçlarından daha küçük olması, sürtünmeye bağlı sıcaklık oluşumunun daha az olmasını sağlamış olabilir. Ayrıca, Super-Snap Rainbow Süper-Cilalama Diski'nin, çalışmada kullanılan silindir ve lastik şeklindeki cilalama uçlarına göre daha düşük pulpal sıcaklık değerleri ortaya çıkarmış olmasının sebebi, diskin ağırlığı ve stabilitesinin diğer uçlara göre daha az olması nedeniyle teması sağlayan uygulama kuvvetine ters kuvvet oluşması ve diskin temas sürekliliğini azaltması olabilir.

Çalışmamızda, 3 farklı firmanın, farklı tip bitirme ve cilalama ürünleri kuru ortamda 10,000 rpm devir ve 0,4 $\mathrm{N}$ basınç ile 10 sn boyunca süt dişi rezin kompozit restorasyonları üzerine uygulandı ve pulpal sıcaklık artışı pulpal kan mikrosirkülasyon modeli (PKMM) yardımıyla belirlendi. En yüksek sıcaklık artışı değeri $2,3^{\circ} \mathrm{C}$ ile Enhance bitim silindiri ve Sof-Lex spiral bitim lastiği gruplarında, en yüksek ortalama sıcaklık ise $1,9 \pm 0,25^{\circ} \mathrm{C}$ ile Sof-Lex spiral bitim lastiği grubunda oldu. Bu değerler, Zach ve Cohen'in [9] klasik çalışmasında gösterilen kritik pulpal sıcaklık artışı olan $5,6^{\circ} \mathrm{C}$ 'nin altındadır. Super-Snap Rainbow Süper-Cilalama diski, tüm gruplar arasında $1,39 \pm 0,3^{\circ} \mathrm{C}$ ile en düşük sıcaklık artışını sağlayan malzeme oldu. Yine de insan süt dişi dentini, daimi diş ve primat dişlerinden farklı özellikler gösterir ve daimi diş ile süt dişini karşılaştıran çalışmalara intiyaç vardır.

Çalışma limitleri dahilinde, Enhance bitim silindiri, Sof-Lex spiral bitim lastiği, Super-Snap Rainbow Bitim Diski, Enhance PoGo cilalama silindiri, Sof-Lex spiral cilalama lastiği ve SuperSnap Rainbow Süper-Cilalama diskinin üretici tarafından önerilen devir ve basınç değerleri dikkate alınarak kısa süreli kullanıldığında süt dişi pulpası için güvenli olduğu söylenebilir. Farklı devir, basınç ve süre değerleriyle daha ileri çalışmalar yapılabilir.

Çıkar ilişkisi: Yazarlar çıkar ilişsisi olmadığını beyan eder.

\section{Kaynaklar}

1. Sahbaz C, Bahsi E, Ince B, Bakir EP, Cellik O. Effect of the different finishing and polishing procedures on the surface roughness of three different posterior composite resins. Scanning 2016;38:448-454. https:// doi.org/10.1002/sca.21295

2. Da Costa J, Ferracane J, Paravina RD, Mazur RF, Roeder L. The effect of different polishing systems on surface roughness and gloss of various resin composites. J Esthet Restor Dent 2007;19:214-224. https://doi.org/10.1111/j.1708-8240.2007.00104.x

3. Alves da Costa GF, de Carvalho Justo Fernandes ACB, Carvalho LAO, de Andrade AC, de Assuncao IV, Borges BCD. Effect of additional polishing methods on the physical surface properties of different nanocomposites: SEM and AFM study. Microsc Res Tech 2018;81:1467-1473. https://doi.org/10.1002/ jemt.23147

4. Bansal K, Gupta S, Nikhil V, Jaiswal S, Jain A, Aggarwal $\mathrm{N}$. Effect of different finishing and polishing systems on the surface roughness of resin composite and enamel: an in vitro profilometric and scanning electron microscopy study. Int J Appl Basic Med Res 2019;9:154-158. https://doi.org/10.4103/ijabmr. ijabmr_11_19

5. Trowbridge HO, Kim S. Pulp development, structure and function. In: Cohen S, Burns RC, ed. 6th ed. Pathways of the Pulp, sixth ed. St. Louis: Mosby, 1994;296-336. Available at: https://www.academia. edu/28876240/Pathways_of_the_pulp_COHEN_. Accessed October 6, 2020

6. Ozturk B, Usumez A, Ozturk AN, Ozer F. In vitro assessment of temperature change in the pulp chamber during cavity preparation. J Prosthet Dent 2004;91:436440. https://doi.org/10.1016/j.prosdent.2004.02.022

7. Martins GR, Cavalcanti BN, Rode SM. Increases in intrapulpal temperature during polymerization of composite resin. J Prosthet Dent 2006;96:328-331. https://doi.org/10.1016/j.prosdent.2006.09.008

8. Dodge WW, Dale RA, Cooley RL, Duke ES. Comparison of wet and dry finishing of resin composites with aluminum oxide discs. Dent Mater 1991;7:18-20. https://doi.org/10.1016/0109-5641(91)90020-Y

9. Zach L, Cohen G. Pulp response to externally applied heat. Oral Surg Oral Med Oral Pathol Oral Radiol 1965;19:515-530. https://doi.org/10.1016/00304220(65) $90015-0$

10. Jakubinek MB, O'Neill C, Felix C, Price RB, White MA. Temperature excursions at the pulp-dentin junction during the curing of light-activated dental restorations. Dent Mater 2008;24:1468-1476. https:// doi.org/10.1016/j.dental.2008.03.012 
11. Kodonas K, Gogos C, Tziafa C. Effect of simulated pulpal microcirculation on intrachamber temperature changes following application of various curing units on tooth surface. J Dent 2009;37:485-490. https://doi. org/10.1016/j.jdent.2009.03.006

12. Uzel A, Buyukyilmaz T, Kayalioglu M, Uzel I. Temperature rise during orthodontic bonding with various light-curing units--an in vitro study. Angle Orthod 2006;76:330-334. https://doi.org/10.1043/00033219(2006)076[0330:TRDOBW]2.0.CO;2

13. Sari T, Celik G, Usumez A. Temperature rise in pulp and gel during laser-activated bleaching: in vitro. Lasers Med Sci 2015;30:577-582. https://doi.org/10.1007/ s10103-013-1375-5

14. Jefferies SR. Abrasive finishing and polishing in restorative dentistry: a state-of-the-art review. Dent Clin North Am 2007;51:379-397. https://doi.org/10.1016/j. cden.2006.12.002

15. Xian C, Shi YY, Lin XJ, Liu D. Experimental study on energy partition of polishing aero-engine blades with abrasive cloth wheel. Int J Adv Manuf Tech 2020;106:1839-1853. https://doi.org/10.1007/s00170019-04690-2

16. Dentistry-adhesion-notched-edge shear bond strength test. International Organization for Standardization. 2013:2. Available at: https://www.iso.org/obp/ ui/\#iso:std:iso:29022:ed-1:v1:en. Accessed October 6, 2020

17. Centers for Disease Control and Prevention. Guidelines for Infection Control in Dental Health-Care Settings - 2003. MMWR 2003;52(No. RR-17):33. Available at: https://www.cdc.gov/mmwr/preview/mmwrhtml/ rr5217a1.htm. Accessed October 6, 2020

18. Ertugrul IF, Orhan EO, Yazkan B. Effect of different dry-polishing regimens on the intrapulpal temperature assessed with pulpal blood microcirculation model. J Esthet Restor Dent 2019;31:268-274. https://doi. org/10.1111/jerd.12442

19. Baik JW, Rueggeberg FA, Liewehr FR. Effect of lightenhanced bleaching on in vitro surface and intrapulpal temperature rise. J Esthet Restor Dent 2001;13:370$378 . \quad$ https://doi.org/10.1111/j.1708-8240.2001. tb01022. $x$

20. Yadav RD, Raisingani D, Jindal D, Mathur R. A comparative analysis of different finishing and polishing devices on nanofilled, microfilled, and hybrid composite: a scanning electron microscopy and profilometric study. Int J Clin Pediatr Dent 2016;9:201208. https://doi.org/10.5005/jp-journals-10005-1364

21. Baldissara $P$, Catapano $S$, Scotti R. Clinical and histological evaluation of thermal injury thresholds in human teeth: a preliminary study. J Oral Rehabil 1997;24:791-801. https://doi. org/10.1111/j.1365-2842.1997.tb00278.x
22. Pohto $M$, Scheinin A. Microscopic observations on living dental pulp II. the effect of thermal irritants on the circulation of the pulp in the lower rat incisor. Acta Odontol Scand 1958;16:315-327. https://doi. org/10.3109/00016355809064116

23. Schubert L. Temperature measurements in teeth using the light beam galvanometer during grinding and drilling. Zahnärztl Welt 1957;58:768-772.

24. Eriksson AR, Albrektsson T. Temperature threshold levels for heat-induced bone tissue injury: a vitalmicroscopic study in the rabbit. J Prosthet Dent 1983;50:101-107. https://doi.org/10.1016/00223913(83)90174-9

25. Hannig M, Bott B. In-vitro pulp chamber temperature rise during composite resin polymerization with various light-curing sources. Dent Mater 1999;15:275-281. https://doi.org/10.1016/S0109-5641(99)00047-0

26. Mollica FB, Camargo FP, Zamboni SC, Pereira SMB, Teixeira SC, Nogueira Junior L. Pulpal temperature increase with high-speed handpiece, Er: YAG laser and ultrasound tips. J Appl Oral Sci 2008;16:209-213. https://doi.org/10.1590/S1678-77572008000300009

27. Cavalcanti BN, Lage Marques JL, Rode SM. Pulpal temperature increases with Er: YAG laser and highspeed handpieces. J Prosthet Dent 2003;90:447-451. https://doi.org/10.1016/j.prosdent.2003.08.022

28. Firoozmand L, Faria R, Araujo MA, di Nicolo R, Huthala MF. Temperature rise in cavities prepared by high and low torque handpieces and $\mathrm{Er}$ : YAG laser. $\mathrm{Br}$ Dent $\mathrm{J}$ 2008;205:1. https://doi.org/10.1038/sj.bdj.2008.491

29. Baldi D, Colombo J, Robiony M, Menini M, Bisagni E, Pera P. Temperature variations in pulp chamber: an in-vitro comparison between ultrasonic and rotating instruments in tooth preparation. Part 1. Minerva Stomatol 2020;69:14-20. https://doi.org/10.23736/ S0026-4970.19.04279-1

30. Baroudi K, Silikas N, Watts DC. In vitro pulp chamber temperature rise from irradiation and exotherm of flowable composites. Int J Paediatr Dent 2009;19:4854. https://doi.org/10.1111/j.1365-263X.2007.00899.x

31. Chiodera G, Gastaldi G, Millar BJ. Temperature change in pulp cavity in vitro during the polymerization of provisional resins. Dent Mater 2009;25:321-325. https://doi.org/10.1016/j.dental.2008.08.006

32. Daronch M, Rueggeberg FA, Hall G, De Goes MF. Effect of composite temperature on in vitro intrapulpal temperature rise. Dent Mater 2007;23:1283-1288. https://doi.org/10.1016/j.dental.2006.11.024

33. Dias M, Choi JJE, Uy CE, Ramani RS, Ganjigatti R, Waddell JN. Real-time pulp temperature change at different tooth sites during fabrication of temporary resin crowns. Heliyon 2019;5:e02971. https://doi. org/10.1016/j.heliyon.2019.e02971 
34. Gubrellay P, Karia M, Talesara K, Sharma C, Raghav S, Sujatha P. Effect of dentin bonding agent on intrapulpal temperature during fabrication of provisional restorations by a direct method: an in vitro study. J Contemp Dent Pract 2019;20:947-951.

35. Ramoglu SI, Karamehmetoglu H, Sari T, Usumez S. Temperature rise caused in the pulp chamber under simulated intrapulpal microcirculation with different light-curing modes. Angle Orthod 2015;85:381-385. https://doi.org/10.2319/030814-164.1

36. Silva PCG, De Fatima Zanirato Lizarelli R, Moriyama LT, De Toledo Porto Neto S, Bagnato VS. Temperature analysis during bonding of brackets using LED or halogen light base units. Photomed Laser Surg 2005;23:41-46. https://doi.org/10.1089/pho.2005.23.41

37. Carrasco TG, Carrasco Guerisoli LD, Froner IC. In vitro study of the pulp chamber temperature rise during lightactivated bleaching. J Appl Oral Sci 2008;16:355-359. http://dx.doi.org/10.1590/S1678-77572008000500010

38. Kivanc BH, Arisu HD, Ulusoy OIA, Saglam BC, Gorgul G. Effect of light-activated bleaching on pulp chamber temperature rise: an in vitro study. Aust Endod J 2012;38:76-79. https://doi.org/10.1111/j.17474477.2010.00271.x

39. Kabbach W, Zezell DM, Pereira TM, Albero FG, Clavijo VRG, de Andrade MF. A thermal investigation of dental bleaching in vitro. Photomed Laser Surg 2008;26:489493. https://doi.org/10.1089/pho.2007.2221

40. Mank S, Steineck M, Brauchli L. Influence of various polishing methods on pulp temperature: an in vitro study. J Orofac Orthop 2011;72:348-357. https://doi. org/10.1007/s00056-011-0039-y

41. Jones CS, Billington RW, Pearson GJ. The effects of lubrication on the temperature rise and surface finish of amalgam and composite resin. J Dent 2007;35:36-42. https://doi.org/10.1016/j.jdent.2006.04.006

42. Briseno B, Ernst CP, Willershausen Zonnchen B. Rise in pulp temperature during finishing and polishing of resin composite restorations: an in vitro study. Quintessence Int 1995;26:361-365.

43. Cobb DS, Dederich DN, Gardner TV. In vitro temperature change at the dentin/pulpal interface by using conventional visible light versus argon laser. Lasers Surg Med 2000;26:386-397. https://doi. org/10.1002/(SICI)1096-9101(2000)26:4<386::AIDLSM7>3.0.CO;2-C

44. Enhance ${ }^{\circledR} \&$ Enhance ${ }^{\circledR}$ mini Finishing Systems. $E$ Enhance ${ }^{\circledR} \mathrm{PoGo}^{\circledR}$ Polishing System. Available at: https://assets.dentsplysirona.com/flagship/en/explore/ restorative/enhance/524357\%20Enhance $\% 20$ mini\%20-\%20multi_WEB\%20final.pdf. Accessed August 17, 2020

45. Shofu product catalogue. Available at: https://www. shofu.de/wp-content/uploads/2019/03/SHOFUKatalog-UK-2019-02.pdf. Accessed August 17, 2020
46. Sof-Lex ${ }^{\mathrm{TM}}$ Spiral Finishing and Polishing System. Technical data sheet. Available at: https://nanopdf. com/download/technical-data-sheet-5b31daa08948f_ pdf. Accessed August 17, 2020

Etik kurul onayı: Pamukkale Üniversitesi Tibbi Etik Kurulu'ndan 18.04.2018 tarih ve 27536 sayı ile onay alınmıştır.

\section{Yazarların makaleye olan katkıları}

Y.E. çalışmanın ana fikrini ve hipotezini kurgulamıştır. Y.E. ve I.F.E. teoriyi geliştirmiş, gereç ve yöntem bölümünü düzenlemişlerdir. Sonuçlar kısmındaki verilerin değerlendirmesini Y.E. ve I.F.E. yapmışlardır. Makalenin tartışma bölümü Y.E. tarafından yazılmış, İ.F.E. gözden geçirip gerekli düzeltmeleri yapmış ve onaylamıştır. Ayrıca tüm yazarlar çalışmanın tamamını tartışmış ve son halini onaylamıştır. 